Provided for non-commercial research and education use. Not for reproduction, distribution or commercial use.

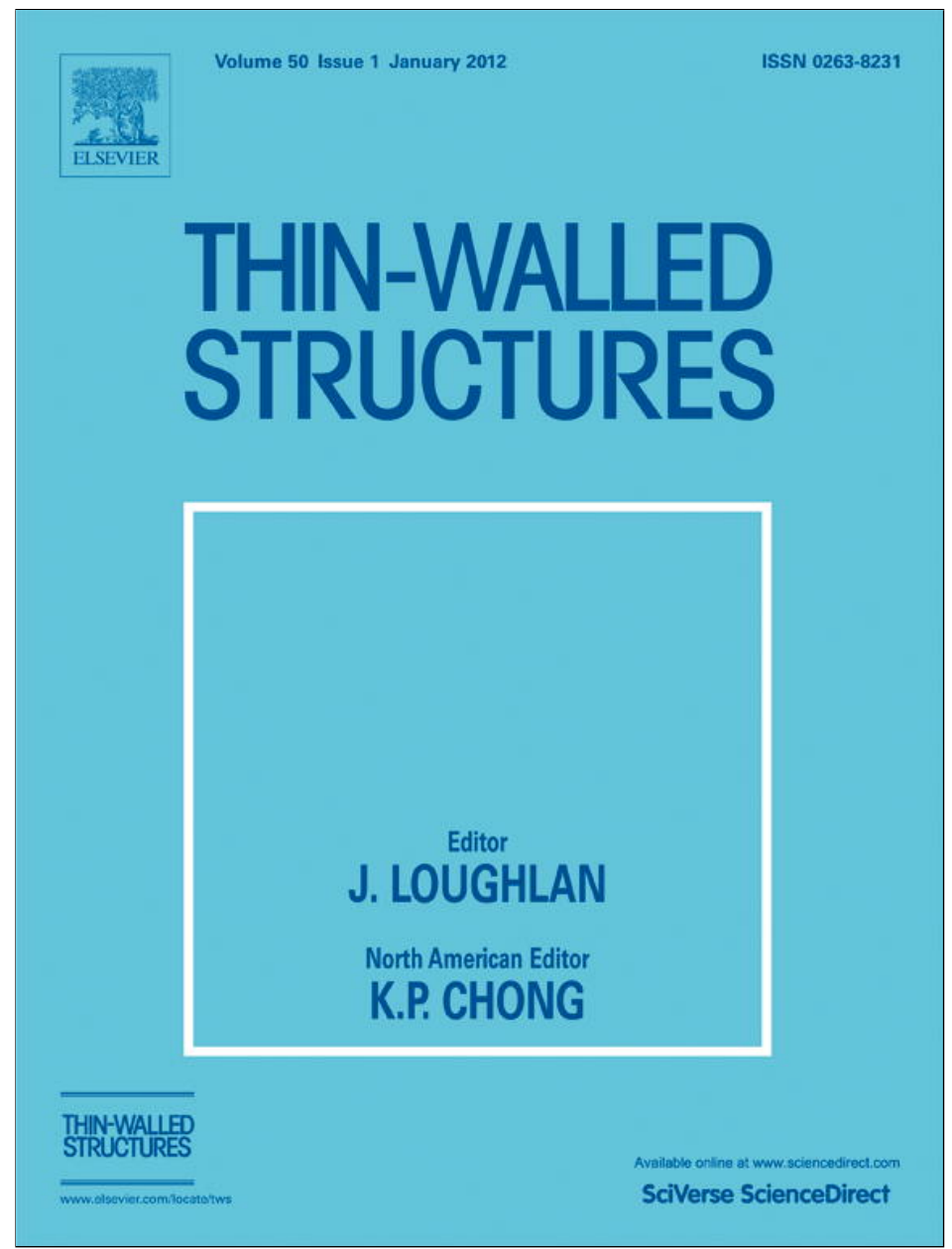

(This is a sample cover image for this issue. The actual cover is not yet available at this time.)

This article appeared in a journal published by Elsevier. The attached copy is furnished to the author for internal non-commercial research and education use, including for instruction at the authors institution and sharing with colleagues.

Other uses, including reproduction and distribution, or selling or licensing copies, or posting to personal, institutional or third party websites are prohibited.

In most cases authors are permitted to post their version of the article (e.g. in Word or Tex form) to their personal website or institutional repository. Authors requiring further information regarding Elsevier's archiving and manuscript policies are encouraged to visit:

http://www.elsevier.com/copyright 


\title{
Cold-formed steel sections with web openings subjected to web crippling under two-flange loading conditions-Part II: Parametric study and proposed design equations
}

\author{
Asraf Uzzaman ${ }^{\mathrm{a}, *}$, James B.P Lim ${ }^{\mathrm{b}}$, David Nash ${ }^{\mathrm{a}}$, Jim Rhodes ${ }^{\mathrm{a}}$, Ben Young ${ }^{\mathrm{c}}$ \\ a Department of Mechanical and Aerospace Engineering, The University of Strathclyde, 75 Montrose Street, Glasgow G1 1XJ, UK \\ ${ }^{\mathrm{b}}$ SPACE, David Keir Building, Queen's University Belfast, Belfast BT9 5AG, UK \\ ${ }^{\mathrm{C}}$ Department of Civil Engineering, The University of Hong Kong, Pokfulam Road, Hong Kong, China
}

\section{A R T I C L E I N F O}

Article history:

Received 12 February 2012

Accepted 12 March 2012

\section{Keywords:}

Cold-formed steel

Web crippling

Finite element analysis

Web hole

Channel section

Reduction factor

Design recommendations

\begin{abstract}
A B S T R A C T
A parametric study of cold-formed steel sections with web openings subjected to web crippling was undertaken using finite element analysis, to investigate the effects of web holes and cross-section sizes on the web crippling strengths of channel sections subjected to web crippling under both interior-twoflange (ITF) and end-two-flange (ETF) loading conditions. In both loading conditions, the hole was centred beneath the bearing plate. It was demonstrated that the main factors influencing the web crippling strength are the ratio of the hole depth to the flat depth of the web, and the ratio of the length of bearing plates to the flat depth of the web. In this paper, design recommendations in the form of web crippling strength reduction factors are proposed, that are conservative to both the experimental and finite element results.
\end{abstract}

(c) 2012 Elsevier Ltd. All rights reserved.

\section{Introduction}

Most design specifications for cold-formed steel structural members provide design rules for cold-formed steel channel sections without web holes; only in the case of the North American Specification for cold-formed steel sections [1] are reduction factors for web crippling with holes presented, covering the cases of interior-oneflange (IOF) and end-one-flange loading (EOF), and with the flanges of the sections unfastened to the support. In addition, in the North American Sepcification, the holes are assumed to be located at the mid-height of the specimen having a longitudinal clear offset distance between the edge of the bearing plates and the web hole.

Web crippling strength reduction factors for cold-formed steel sections under interior-one-flange (IOF) and end-one-flange loading (EOF) have been developed by Yu and Davis [2], Sivakumaran and Zielonka [3] LaBoube et al. [4], and Chung [5]. The authors have also developed web crippling strength reduction factors for cold-formed steel sections with offset web holes subjected to the interior-twoflange (ITF) loading condition (Uzzaman et al. [6]). Zhou and Young [7] have recommended web crippling strength reduction factors of aluminium alloy square hollow sections under ITF and ETF loading conditions. However, no design recommendations are available for cold-formed steel sections with web opening subjected to web

\footnotetext{
* Corresponding author. Tel.: +44 787768785.

E-mail address: asraf.uzzaman@strath.ac.uk (A. Uzzaman).
}

crippling under ITF and ETF loading conditions, where the opening is located beneath the bearing plates.

Experimental and numerical investigations have been discussed in the companion paper [8]. In this paper, non-linear elasto-plastic finite element analyses (FEA) are used to conduct parametric studies to investigate the effect of circular web holes on the web crippling strength of lipped channel sections for the interior-two-flange (ITF) and end-two-flange (ETF) loading conditions, as shown in Fig. 1. The cases of both flanges fastened and unfastened to the support are considered. In both loading conditions, the hole was centred beneath the bearing plate. The general purpose finite element program ANSYS [9] was used for the parametric study. Based on the test data found in Uzzaman et al. [8] and the numerical results obtained from this study an extensive statistics analysis is performed. Design recommendations in the form of web crippling strength reduction factors are proposed for ITF and ETF loading conditions, that are conservative to both the experimental and finite element results.

\section{Experiment investigation}

Uzzaman et al. [8] conducted a series of test programmes on lipped channel sections, as shown in Fig. 1, with circular web holes subjected to web crippling under ITF and ETF loading conditions. The cases of both flanges fastened and flanges 


\begin{tabular}{|c|c|c|c|}
\hline \multicolumn{2}{|c|}{ Nomenclature } & \multirow{2}{*}{$\begin{array}{l}P_{\text {EXP }} \\
P_{\text {FEA }}\end{array}$} & \multirow{2}{*}{$\begin{array}{l}\text { Experimental ultimate web crippling load per web; } \\
\text { Web crippling strength per web predicted from finite } \\
\text { element (FEA); }\end{array}$} \\
\hline$A$ & Web holes ratio; & & \\
\hline$a$ & Diameter of circular web holes; & $P_{\mathrm{BS}}$ & Nominal web crippling strength obtained from British \\
\hline$b_{f}$ & Overall flange width of section; & & Standard; \\
\hline$b_{l}$ & Overall lip width of section; & $P_{\text {Euro }}$ & Nominal web crippling strength obtained from \\
\hline $\mathrm{COV}$ & Coefficient of variation; & & European code; \\
\hline $\mathrm{DL}$ & Dead load; & $P_{\mathrm{NAS}}$ & Nominal web crippling strength obtained from North \\
\hline$D$ & Overall web depth of section; & & American Specification; \\
\hline E & Young's modulus of elasticity; & $P_{m}$ & Mean value of tested-to-predicted load ratio; \\
\hline FEA & Finite element analysis; & $R$ & Reduction factor; \\
\hline$F_{m}$ & Mean value of fabrication factor; & $R_{P}$ & Proposed reduction factor; \\
\hline$f_{\mathrm{y}}^{m}$ & Material yield strength; & $r_{i}$ & Inside corner radius of section; \\
\hline$h$ & Depth of the flat portion of web; & $t$ & Thickness of section; \\
\hline$L$ & Length of the specimen; & $V_{F}$ & Coefficient of variation of fabrication factor; \\
\hline LL & Live load; & $V_{M}$ & Coefficient of variation of material factor; \\
\hline$M_{m}$ & Mean value of material factor; & $V_{P}$ & Coefficient of variation of tested-to-predicted \\
\hline$N$ & Length of the bearing plate; & & load ratio; \\
\hline$P$ & Experimental and finite element ultimate web & $\beta$ & Reliability index; \\
\hline & crippling load per web; & $\phi$ & Resistance factor; \\
\hline
\end{tabular}

unfastened to the support were considered. The size of the web holes was varied in order to investigate the effect of the web holes on the web crippling strength. Circular holes with nominal diameters (a) ranging from $40 \mathrm{~mm}$ to $240 \mathrm{~mm}$ were considered in the experimental investigation. The ratio of the diameter of the holes to the depth of the flat portion of the webs $(a / h)$ were 0.2 , $0.4,0.6$ and 0.8 . All the test specimens were fabricated with web holes located at the mid-depth of the webs and centred beneath the bearing plates. The test data reported in the companion paper [8] are used in this paper for the development of web crippling strength reduction factor equations Fig. 2 .

\section{Numerical investigation}

The non-linear elasto-plastic general purpose finite element program ANSYS [9] was used to simulate the channel sections with and without holes subjected to web crippling. An accurate and reliable non-linear FEM for the channel sections has been presented by Uzzaman et al. [8]. The details of the FEM are described in Uzzaman et al. [8]. In the finite element model, the measured cross-

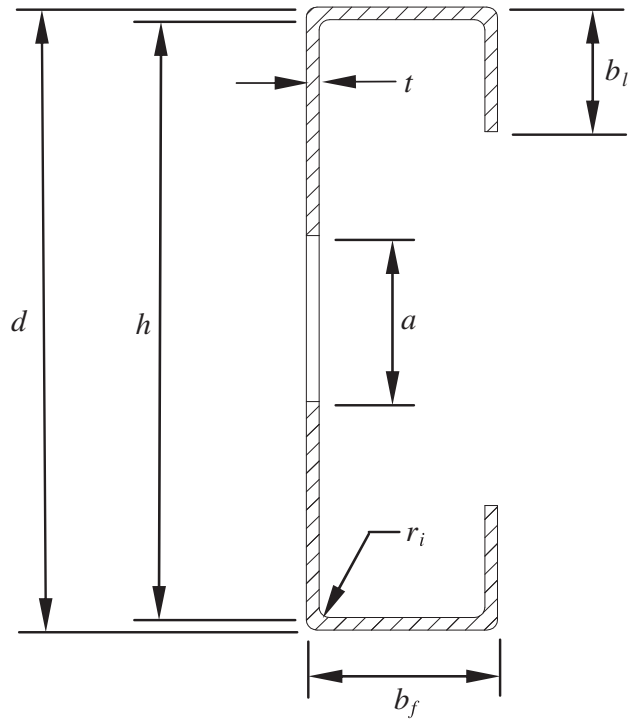

Fig. 2. Definition of symbols.
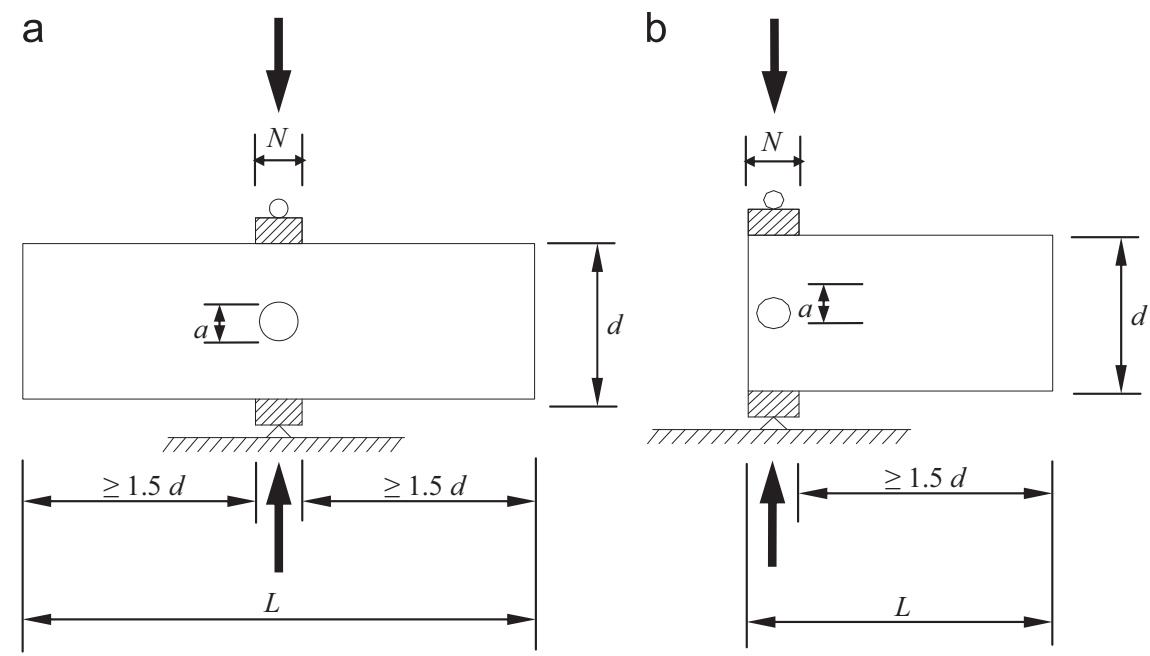

Fig. 1. Loading condition. (a) ITF loading condition (b) ETF loading condition. 
section dimensions and the material properties obtained from the tests were used. The model was based on the centreline dimensions of the cross-sections. The material non-linearity was incorporated in the finite element model by specifying 'true' values of stresses and strains. The plasticity of the material was determined by a mathematical model, known as the incremental plasticity model. Depending on the size of the section, the finite element mesh sizes ranged from $3 \times 3 \mathrm{~mm}$ (length by width) to $5 \times 5 \mathrm{~mm}$. The bearing plates, the channel section with circular holes and the interfaces between the bearing plates and the channel section have been modelled. Contact surfaces were defined between the bearing plate and the cold-formed steel section.

\section{Parametric study}

The finite element model developed closely predicted the experimental ultimate loads and failure modes of the channel sections with and without circular web holes subjected to web crippling [8]. Using this model, parametric studies were carried out to study the effects of web holes and cross-section sizes on the web crippling strengths of channel sections subjected to web crippling under ITF and ETF loading conditions. The cases of both flange fastened and flange unfastened to the support were considered. The web holes were located at the mid-depth of the webs and centred beneath the bearing plates. The web crippling strength predicted was influenced primarily by the ratio of the hole depth to the flat portion of the web, $a / h$, and the ratio of the bearing length to the flat portion of the web $N / h$. In order to find the effect of $a / h$ and $N / h$ on web crippling strength considering web holes, parametric studies were carried out considering the web holes, the cross-section sizes and the different bearing plate lengths.

The specimens consisted of two different section sizes, having thicknesses $(t)$ ranging from $1.4 \mathrm{~mm}$ to $6.0 \mathrm{~mm}$ and web slenderness $(h / t)$ value ranging from 31.8 to 176.9 . The ratios of the diameter of the holes $(a)$ to the depth of the flat portion of the webs $(h)$ were $0.2,0.4,0.6$ and 0.8 . Bearing plates of lengths $(N)$

Table 1

Dimensions and web crippling strength predicted from FEA of parametric study of $a / h$ for flanges unfastened under ITF loading condition.

\begin{tabular}{|c|c|c|c|c|c|c|c|c|c|c|}
\hline \multirow[t]{2}{*}{ Specimen } & \multirow{2}{*}{$\begin{array}{l}\text { Web } \\
d \\
(\mathrm{~mm})\end{array}$} & \multirow{2}{*}{$\begin{array}{l}\text { Flange } \\
b_{f} \\
(\mathrm{~mm})\end{array}$} & \multirow{2}{*}{$\begin{array}{l}\text { Lip } \\
b_{l} \\
(\mathrm{~mm})\end{array}$} & \multirow{2}{*}{$\begin{array}{l}\text { Thickness } \\
t \\
(\mathrm{~mm})\end{array}$} & \multirow{2}{*}{$\begin{array}{l}\text { Length } \\
L \\
(\mathrm{~mm})\end{array}$} & \multicolumn{5}{|c|}{ FEA load per web, $P_{\mathrm{FEA}}$} \\
\hline & & & & & & $\begin{array}{l}\mathrm{A} 0 \\
(\mathrm{kN})\end{array}$ & $\begin{array}{l}\mathrm{A} 0.2 \\
(\mathrm{kN})\end{array}$ & $\begin{array}{l}\mathrm{A} 0.4 \\
(\mathrm{kN})\end{array}$ & $\begin{array}{l}\mathrm{A} 0.6 \\
(\mathrm{kN})\end{array}$ & $\begin{array}{l}\mathrm{A} 0.8 \\
(\mathrm{kN})\end{array}$ \\
\hline ITF202 $\times 65 \times 13-\mathrm{t} 1.4 \mathrm{~N} 100 \mathrm{FR}$ & 202.5 & 63.6 & 16.0 & 1.40 & 736.4 & 18.4 & 17.4 & 15.7 & - & - \\
\hline ITF202 $\times 65 \times 13-\mathrm{t} 4.0 \mathrm{~N} 100 \mathrm{FR}$ & 202.5 & 63.6 & 16.0 & 4.00 & 736.4 & 81.8 & 76.0 & 66.2 & - & - \\
\hline ITF202 $\times 65 \times 13-$ t6.0N100FR & 202.5 & 63.6 & 16.0 & 6.00 & 736.4 & 176.3 & 163.0 & 142.0 & - & - \\
\hline ITF202 $\times 65 \times 13-\mathrm{t} 1.4$ N150FR & 202.5 & 64.0 & 17.5 & 1.44 & 786.4 & 19.3 & 18.2 & 16.5 & 14.2 & - \\
\hline ITF202 $\times 65 \times 13-\mathrm{t} 4.0 \mathrm{~N} 150 \mathrm{FR}$ & 202.5 & 64.0 & 17.5 & 4.00 & 786.4 & 84.4 & 78.9 & 69.8 & 58.9 & - \\
\hline ITF202 $\times 65 \times 13-$ t6.0N150FR & 202.5 & 64.0 & 17.5 & 6.00 & 786.4 & 183.0 & 170.6 & 151.0 & 129.2 & - \\
\hline ITF202 $\times 65 \times 13-\mathrm{t} 1.4 \mathrm{~N} 200 \mathrm{FR}$ & 202.5 & 64.0 & 17.5 & 1.44 & 836.4 & 20.2 & 19.1 & 17.5 & 15.3 & 12.2 \\
\hline ITF202 $\times 65 \times 13-\mathrm{t} 4.0 \mathrm{~N} 200 \mathrm{FR}$ & 202.5 & 64.0 & 17.5 & 4.00 & 836.4 & 87.5 & 82.1 & 73.9 & 63.8 & 54.1 \\
\hline ITF202 × $65 \times 13-$ t6.0N200FR & 202.5 & 64.0 & 17.5 & 6.00 & 836.4 & 190.0 & 178.0 & 160.2 & 139.2 & 120.4 \\
\hline ITF302 × $90 \times 18$-t2N150FR & 304.0 & 87.9 & 18.0 & 2.00 & 1112.2 & 15.0 & 14.5 & 13.4 & - & - \\
\hline ITF302 $\times 90 \times 18-t 4.0$ N150FR & 304.0 & 87.9 & 18.0 & 4.00 & 1112.2 & 80.5 & 75.1 & 65.6 & - & - \\
\hline ITF302 × $90 \times 18$-t6.0N150FR & 304.0 & 87.9 & 18.0 & 6.00 & 1112.2 & 184.3 & 170.6 & 147.9 & - & - \\
\hline ITF302 × $90 \times 18$-t2N220FR & 303.8 & 88.8 & 18.7 & 2.00 & 1182.2 & 15.9 & 15.3 & 14.2 & 12.8 & - \\
\hline ITF302 $\times 90 \times 18-$ t4.0N220FR & 303.8 & 88.8 & 18.7 & 4.00 & 1182.2 & 83.2 & 77.8 & 68.8 & 57.4 & - \\
\hline ITF302 $\times 90 \times 18$-t6.0N220FR & 303.8 & 88.8 & 18.7 & 6.00 & 1182.2 & 190.0 & 176.9 & 155.6 & 130.3 & - \\
\hline ITF302 × $90 \times 18$-t2N300FR & 303.8 & 88.8 & 18.7 & 2.00 & 1262.2 & 16.9 & 16.3 & 15.2 & 13.7 & 10.9 \\
\hline ITF302 $\times 90 \times 18-\mathrm{t} 4.0$ N300FR & 303.8 & 88.8 & 18.7 & 4.00 & 1262.2 & 86.6 & 81.2 & 73.0 & 62.8 & 51.4 \\
\hline ITF302 × $90 \times 18$-t6.0N300FR & 303.8 & 88.8 & 18.7 & 6.00 & 1262.2 & 197.7 & 184.7 & 165.1 & 142.1 & 120.1 \\
\hline
\end{tabular}

Table 2

Dimensions and web crippling strength predicted from FEA of parametric study of $a / h$ for flanges fastened under ITF loading condition.

\begin{tabular}{|c|c|c|c|c|c|c|c|c|c|c|}
\hline \multirow[t]{2}{*}{ Specimen } & \multirow{2}{*}{$\begin{array}{l}\text { Web } \\
d \\
(\mathrm{~mm})\end{array}$} & \multirow{2}{*}{$\begin{array}{l}\text { Flange } \\
b_{f} \\
(\mathrm{~mm})\end{array}$} & \multirow{2}{*}{$\begin{array}{l}\text { Lip } \\
b_{l} \\
(\mathrm{~mm})\end{array}$} & \multirow{2}{*}{$\begin{array}{l}\text { Thickness } \\
t \\
(\mathrm{~mm})\end{array}$} & \multirow{2}{*}{$\begin{array}{l}\text { Length } \\
L \\
(\mathrm{~mm})\end{array}$} & \multicolumn{5}{|c|}{ FEA load per web, $P_{\mathrm{FEA}}$} \\
\hline & & & & & & $\begin{array}{l}\mathrm{A} 0 \\
(\mathrm{kN})\end{array}$ & $\begin{array}{l}\mathrm{A} 0.2 \\
(\mathrm{kN})\end{array}$ & $\begin{array}{l}\mathrm{A} 0.4 \\
(\mathrm{kN})\end{array}$ & $\begin{array}{l}\mathrm{A} 0.6 \\
(\mathrm{kN})\end{array}$ & $\begin{array}{l}\mathrm{A} 0.8 \\
(\mathrm{kN})\end{array}$ \\
\hline ITF202 $\times 65 \times 13-\mathrm{t} 1.4 \mathrm{~N} 100 \mathrm{FX}$ & 202.5 & 63.6 & 16.0 & 1.4 & 736.4 & 26.7 & 26.0 & 22.9 & - & - \\
\hline ITF202 $\times 65 \times 13-t 4.0$ N100FX & 202.5 & 63.6 & 16.0 & 4.0 & 736.4 & 99.6 & 97.6 & 88.5 & - & - \\
\hline ITF202 $\times 65 \times 13-\mathrm{t} 6.0 \mathrm{~N} 100 \mathrm{FX}$ & 202.5 & 63.6 & 16.0 & 6.0 & 736.4 & 207.7 & 206.0 & 178.3 & - & - \\
\hline ITF202 $\times 65 \times 13-t 1.4$ N150FX & 202.5 & 64.0 & 17.5 & 1.4 & 786.4 & 28.4 & 27.0 & 24.2 & 20.6 & - \\
\hline ITF202 $\times 65 \times 13-\mathrm{t} 4.0 \mathrm{~N} 150 \mathrm{FX}$ & 202.5 & 64.0 & 17.5 & 4.0 & 786.4 & 114.1 & 107.2 & 95.0 & 82.0 & - \\
\hline ITF202 $\times 65 \times 13-\mathrm{t} 6.0 \mathrm{~N} 150 \mathrm{FX}$ & 202.5 & 64.0 & 17.5 & 6.0 & 786.4 & 232.2 & 218.7 & 195.3 & 170.7 & - \\
\hline ITF202 $\times 65 \times 13-\mathrm{t} 1.4 \mathrm{~N} 200 \mathrm{FX}$ & 202.5 & 64.0 & 17.5 & 1.4 & 836.4 & 29.6 & 28.1 & 25.6 & 22.4 & 19.0 \\
\hline ITF202 $\times 65 \times 13-\mathrm{t} 4.0 \mathrm{~N} 200 \mathrm{FX}$ & 202.5 & 64.0 & 17.5 & 4.0 & 836.4 & 119.3 & 112.7 & 101.9 & 89.7 & 78.1 \\
\hline ITF202 $\times 65 \times 13-\mathrm{t} 6.0 \mathrm{~N} 200 \mathrm{FX}$ & 202.5 & 64.0 & 17.5 & 6.0 & 836.4 & 245.9 & 232.9 & 212.2 & 189.6 & 167.4 \\
\hline ITF302 $\times 90 \times 18-t 2 N 150 F X$ & 304.0 & 87.9 & 18.0 & 2.0 & 1112.2 & 24.5 & 23.7 & 21.3 & - & - \\
\hline ITF302 $\times 90 \times 18-$ t4.0N150FX & 304.0 & 87.9 & 18.0 & 4.0 & 1112.2 & 111.1 & 104.7 & 91.1 & - & - \\
\hline ITF302 $\times 90 \times 18$-t6.0N150FX & 304.0 & 87.9 & 18.0 & 6.0 & 1112.2 & 233.7 & 225.1 & 195.0 & - & - \\
\hline ITF302 × $90 \times 18-t 2 N 220 F X$ & 303.8 & 88.8 & 18.7 & 2.0 & 1182.2 & 25.6 & 24.5 & 22.2 & 18.9 & - \\
\hline ITF302 $\times 90 \times 18-t 4.0 N 220 F X$ & 303.8 & 88.8 & 18.7 & 4.0 & 1182.2 & 115.5 & 108.5 & 96.1 & 81.9 & - \\
\hline ITF302 $\times 90 \times 18-$ t6.0N220FX & 303.8 & 88.8 & 18.7 & 6.0 & 1182.2 & 250.8 & 234.8 & 207.8 & 179.0 & - \\
\hline ITF302 × $90 \times 18$-t2N300FX & 303.8 & 88.8 & 18.7 & 2.0 & 1262.2 & 26.7 & 25.5 & 23.4 & 20.7 & 17.6 \\
\hline ITF302 $\times 90 \times 18-\mathrm{t} 4.0 \mathrm{~N} 300 \mathrm{FX}$ & 303.8 & 88.8 & 18.7 & 4.0 & 1262.2 & 120.9 & 113.8 & 102.8 & 89.8 & 76.5 \\
\hline ITF302 $\times 90 \times 18-t 6.0 N 300 F X$ & 303.8 & 88.8 & 18.7 & 6.0 & 1262.2 & 264.3 & 248.3 & 224.5 & 197.4 & 170.6 \\
\hline
\end{tabular}


Table 3

Dimensions and web crippling strength predicted from FEA of parametric study of $a / h$ for flanges unfastened under ETF loading condition.

\begin{tabular}{|c|c|c|c|c|c|c|c|c|c|c|}
\hline \multirow[t]{2}{*}{ Specimen } & \multirow{2}{*}{$\begin{array}{l}\text { Web } \\
d \\
(\mathrm{~mm})\end{array}$} & \multirow{2}{*}{$\begin{array}{l}\text { Flange } \\
b_{f} \\
(\mathrm{~mm})\end{array}$} & \multirow{2}{*}{$\begin{array}{l}\text { Lip } \\
b_{l} \\
(\mathrm{~mm})\end{array}$} & \multirow{2}{*}{$\begin{array}{l}\text { Thickness } \\
t \\
(\mathrm{~mm})\end{array}$} & \multirow{2}{*}{$\begin{array}{l}\text { Length } \\
L \\
(\mathrm{~mm})\end{array}$} & \multicolumn{5}{|c|}{ FEA load per web, $P_{\mathrm{FEA}}$} \\
\hline & & & & & & $\begin{array}{l}\mathrm{A} 0 \\
(\mathrm{kN})\end{array}$ & $\begin{array}{l}\mathrm{A} 0.2 \\
(\mathrm{kN})\end{array}$ & $\begin{array}{l}\mathrm{A} 0.4 \\
(\mathrm{kN})\end{array}$ & $\begin{array}{l}\mathrm{A} 0.6 \\
(\mathrm{kN})\end{array}$ & $\begin{array}{l}\mathrm{A} 0.8 \\
(\mathrm{kN})\end{array}$ \\
\hline ETF202 $\times 65 \times 13-\mathrm{t} 1.4 \mathrm{~N} 100 \mathrm{FR}$ & 202.5 & 63.6 & 16.0 & 1.40 & 410.0 & 2.9 & 2.5 & 2.1 & - & - \\
\hline ETF202 $\times 65 \times 13-$ t4.0N100FR & 202.5 & 63.6 & 16.0 & 4.00 & 410.0 & 35.2 & 30.0 & 25.2 & - & - \\
\hline ETF202 $\times 65 \times 13-$ t6.0N100FR & 202.5 & 63.6 & 16.0 & 6.00 & 410.0 & 80.1 & 67.8 & 57.3 & - & - \\
\hline ETF202 $\times 65 \times 13-\mathrm{t} 1.4 \mathrm{~N} 150 \mathrm{FR}$ & 202.5 & 64.0 & 17.5 & 1.44 & 460.0 & 3.2 & 2.8 & 2.4 & 1.9 & - \\
\hline ETF202 $\times 65 \times 13-\mathrm{t} 4.0 \mathrm{~N} 150 \mathrm{FR}$ & 202.5 & 64.0 & 17.5 & 4.00 & 460.0 & 43.0 & 37.4 & 31.9 & 26.8 & - \\
\hline ETF202 $\times 65 \times 13-\mathrm{t} 6.0 \mathrm{~N} 150 \mathrm{FR}$ & 202.5 & 64.0 & 17.5 & 6.00 & 460.0 & 95.8 & 83.5 & 72.2 & 61.3 & - \\
\hline ETF202 $\times 65 \times 13-\mathrm{t} 1.4 \mathrm{~N} 200 \mathrm{FR}$ & 202.5 & 64.0 & 17.5 & 1.44 & 510.0 & 3.9 & 3.5 & 3.0 & 2.6 & 2.2 \\
\hline ETF202 $\times 65 \times 13-$ t4.0N200FR & 202.5 & 64.0 & 17.5 & 4.00 & 510.0 & 50.9 & 45.2 & 39.5 & 33.9 & 28.4 \\
\hline ETF202 $\times 65 \times 13-$ t6.0N200FR & 202.5 & 64.0 & 17.5 & 6.00 & 510.0 & 111.9 & 99.1 & 87.4 & 75.8 & 64.3 \\
\hline ETF302 × $90 \times 18$-t2N150FR & 304.0 & 87.9 & 18.0 & 2.00 & 662.0 & 5.1 & 4.5 & 3.6 & - & - \\
\hline ETF302 $\times 90 \times 18-$-t4.0N150FR & 304.0 & 87.9 & 18.0 & 4.00 & 662.0 & 33.2 & 28.2 & 23.4 & - & - \\
\hline ETF302 $\times 90 \times 18-$ t6. 0 N150FR & 304.0 & 87.9 & 18.0 & 6.00 & 662.0 & 79.6 & 67.4 & 56.3 & - & - \\
\hline ETF302 × $90 \times 18$-t2N220FR & 303.8 & 88.8 & 18.7 & 2.00 & 732.0 & 6.4 & 5.6 & 4.7 & 3.8 & - \\
\hline ETF302 $\times 90 \times 18-$ t4 .0 N220FR & 303.8 & 88.8 & 18.7 & 4.00 & 732.0 & 40.0 & 34.5 & 29.0 & 24.0 & - \\
\hline ETF302 $\times 90 \times 18$-t6.0N220FR & 303.8 & 88.8 & 18.7 & 6.00 & 732.0 & 94.8 & 81.8 & 69.4 & 56.9 & - \\
\hline ETF302 × $90 \times 18$-t2N300FR & 303.8 & 88.8 & 18.7 & 2.00 & 812.0 & 7.9 & 7.1 & 6.1 & 5.1 & 4.1 \\
\hline ETF302 $\times 90 \times 18-$-t4.0N300FR & 303.8 & 88.8 & 18.7 & 4.00 & 812.0 & 48.2 & 42.7 & 36.9 & 31.2 & 25.8 \\
\hline ETF302 $\times 90 \times 18-$-t6.0N300FR & 303.8 & 88.8 & 18.7 & 6.00 & 812.0 & 112.5 & 99.3 & 86.6 & 74.1 & 61.9 \\
\hline
\end{tabular}

Table 4

Dimensions and web crippling strength predicted from FEA of parametric study of $a / h$ for flanges fastened under ETF loading condition.

\begin{tabular}{|c|c|c|c|c|c|c|c|c|c|c|}
\hline \multirow[t]{2}{*}{ Specimen } & \multirow{2}{*}{$\begin{array}{l}\text { Web } \\
d \\
(\mathrm{~mm})\end{array}$} & \multirow{2}{*}{$\begin{array}{l}\text { Flange } \\
b_{f} \\
(\mathrm{~mm})\end{array}$} & \multirow{2}{*}{$\begin{array}{l}\text { Lip } \\
b_{l} \\
(\mathrm{~mm})\end{array}$} & \multirow{2}{*}{$\begin{array}{l}\text { Thickness } \\
t \\
(\mathrm{~mm})\end{array}$} & \multirow{2}{*}{$\begin{array}{l}\text { Length } \\
L \\
(\mathrm{~mm})\end{array}$} & \multicolumn{5}{|c|}{ FEA load per web, $P_{\mathrm{FEA}}$} \\
\hline & & & & & & $\begin{array}{l}\mathrm{A} 0 \\
(\mathrm{kN})\end{array}$ & $\begin{array}{l}\mathrm{A} 0.2 \\
(\mathrm{kN})\end{array}$ & $\begin{array}{l}\mathrm{A} 0.4 \\
(\mathrm{kN})\end{array}$ & $\begin{array}{l}\mathrm{A} 0.6 \\
(\mathrm{kN})\end{array}$ & $\begin{array}{l}\mathrm{A} 0.8 \\
(\mathrm{kN})\end{array}$ \\
\hline ETF202 $\times 65 \times 13-\mathrm{t} 1.4 \mathrm{~N} 100 \mathrm{FX}$ & 202.5 & 63.6 & 16.0 & 1.40 & 410.0 & 5.4 & 4.9 & 4.3 & - & - \\
\hline ETF202 $\times 65 \times 13-\mathrm{t} 4.0 \mathrm{~N} 100 \mathrm{FX}$ & 202.5 & 63.6 & 16.0 & 4.00 & 410.0 & 52.4 & 46.6 & 41.2 & - & - \\
\hline ETF202 $\times 65 \times 13-\mathrm{t} 6.0 \mathrm{~N} 100 \mathrm{FX}$ & 202.5 & 63.6 & 16.0 & 6.00 & 410.0 & 112.3 & 98.5 & 86.7 & - & - \\
\hline ETF202 $\times 65 \times 13-t 1.4 N 150 F X$ & 202.5 & 64.0 & 17.5 & 1.44 & 460.0 & 6.0 & 5.6 & 5.0 & 4.4 & - \\
\hline ETF202 $\times 65 \times 13-\mathrm{t} 4.0 \mathrm{~N} 150 \mathrm{FX}$ & 202.5 & 64.0 & 17.5 & 4.00 & 460.0 & 67.1 & 60.4 & 54.1 & 47.9 & - \\
\hline ETF202 $\times 65 \times 13$-t6.0N150FX & 202.5 & 64.0 & 17.5 & 6.00 & 460.0 & 143.9 & 128.7 & 114.9 & 102.2 & - \\
\hline ETF202 $\times 65 \times 13-t 1.4$ N200FX & 202.5 & 64.0 & 17.5 & 1.44 & 510.0 & 7.2 & 6.7 & 6.1 & 5.5 & 4.6 \\
\hline ETF202 $\times 65 \times 13-\mathrm{t} 4.0 \mathrm{~N} 200 \mathrm{FX}$ & 202.5 & 64.0 & 17.5 & 4.00 & 510.0 & 82.0 & 75.4 & 67.9 & 60.3 & 50.8 \\
\hline ETF202 $\times 65 \times 13-\mathrm{t} 6.0 \mathrm{~N} 200 \mathrm{FX}$ & 202.5 & 64.0 & 17.5 & 6.00 & 510.0 & 175.6 & 159.7 & 144.4 & 129.1 & 109.0 \\
\hline ETF302 $\times 90 \times 18$-t2N150FX & 304.0 & 87.9 & 18.0 & 2.00 & 662.0 & 9.8 & 8.9 & 7.7 & - & - \\
\hline ETF302 $\times 90 \times 18$-t4.0N150FX & 304.0 & 87.9 & 18.0 & 4.00 & 662.0 & 53.0 & 47.1 & 41.3 & - & - \\
\hline ETF302 $\times 90 \times 18-t 6.0$ N150FX & 304.0 & 87.9 & 18.0 & 6.00 & 662.0 & 119.1 & 104.6 & 91.8 & - & - \\
\hline ETF302 $\times 90 \times 18-t 2 N 220 F X$ & 303.8 & 88.8 & 18.7 & 2.00 & 732.0 & 12.3 & 11.3 & 10.0 & 8.6 & - \\
\hline ETF302 $\times 90 \times 18$-t4.0N220FX & 303.8 & 88.8 & 18.7 & 4.00 & 732.0 & 65.7 & 59.5 & 52.7 & 46.1 & - \\
\hline ETF302 $\times 90 \times 18$-t6.0N220FX & 303.8 & 88.8 & 18.7 & 6.00 & 732.0 & 148.9 & 133.2 & 117.6 & 103.5 & - \\
\hline ETF302 $\times 90 \times 18$-t2N300FX & 303.8 & 88.8 & 18.7 & 2.00 & 812.0 & 14.9 & 13.9 & 12.6 & 11.2 & 9.2 \\
\hline ETF302 $\times 90 \times 18-\mathrm{t} 4.0 \mathrm{~N} 300 \mathrm{FX}$ & 303.8 & 88.8 & 18.7 & 4.00 & 812.0 & 81.0 & 74.9 & 67.3 & 58.9 & 47.7 \\
\hline ETF302 $\times 90 \times 18-\mathrm{t} 6.0 \mathrm{~N} 300 \mathrm{FX}$ & 303.8 & 88.8 & 18.7 & 6.00 & 812.0 & 183.4 & 167.5 & 150.1 & 132.5 & 109.5 \\
\hline
\end{tabular}

Table 5

Comparison of experimental and numerical results with design strength for ETF and ITF loading condition under flanges unfastened case.

\begin{tabular}{|c|c|c|c|c|c|c|c|c|c|}
\hline \multirow[t]{2}{*}{ Specimen } & \multirow[t]{2}{*}{$h / t$} & \multirow[t]{2}{*}{$N / t$} & \multirow[t]{2}{*}{$N / h$} & \multirow[t]{2}{*}{$r_{i} / t$} & \multirow{2}{*}{$\begin{array}{l}\text { Failure load } \\
\left(P_{\mathrm{EXP}} \text { and } P_{\mathrm{FEA}}\right) \\
P \\
(\mathrm{kN})\end{array}$} & \multicolumn{2}{|c|}{$\begin{array}{l}\text { Web crippling strength predicted from } \\
\text { current design codes }\end{array}$} & \multicolumn{2}{|c|}{ Comparison } \\
\hline & & & & & & $\begin{array}{l}P_{\mathrm{BS}} \\
(\mathrm{kN})\end{array}$ & $\begin{array}{l}P_{\mathrm{EC} 3} \\
(\mathrm{kN})\end{array}$ & $P / P_{\mathrm{BS}}$ & $P / P_{\mathrm{EC} 3}$ \\
\hline ETF142 $\times 60 \times 13-\mathrm{t} 1.3 \mathrm{MN} 90 \mathrm{AOFR}$ & 113.3 & 72.6 & 0.6 & 3.8 & 2.2 & 2.3 & 2.3 & 0.97 & 0.97 \\
\hline ETF142 $\times 60 \times 13$-t1.3MN120AOFR & 113.3 & 96.8 & 0.9 & 3.8 & 2.4 & 2.6 & 2.6 & 0.90 & 0.90 \\
\hline ETF172 $\times 65 \times 13-t 1.3 M N 120 A 0 F R$ & 134.0 & 94.5 & 0.7 & 3.9 & 2.4 & 2.4 & 2.4 & 0.99 & 0.99 \\
\hline ETF202 × $65 \times 13-t 1.4 M N 120 A 0 F R$ & 137.4 & 82.8 & 0.6 & 3.4 & 2.7 & 3.3 & 3.3 & 0.82 & 0.82 \\
\hline ETF202 $\times 65 \times 13-t 1.4 M N 100 A 0 F R$ & 142.3 & 71.4 & 0.5 & 3.6 & 2.9 & 2.8 & 2.8 & 1.06 & 1.06 \\
\hline ETF202 $\times 65 \times 13-t 1.4 M N 150 A 0 F R$ & 142.3 & 107.1 & 0.8 & 3.6 & 3.2 & 3.3 & 3.3 & 0.96 & 0.96 \\
\hline ETF202 $\times 65 \times 13-t 1.4 M N 200 A 0 F R$ & 142.3 & 142.9 & 1.0 & 3.6 & 3.9 & 3.9 & 3.9 & 0.99 & 0.99 \\
\hline ITF142 $\times 60 \times 13-\mathrm{t} 1.3 \mathrm{MN} 90 \mathrm{AOFR}$ & 113.3 & 72.6 & 0.6 & 3.8 & 6.0 & 7.0 & 7.2 & 0.86 & 0.84 \\
\hline ITF142 $\times 60 \times 13-\mathrm{t} 1.3 \mathrm{MN} 120$ A0FR & 113.3 & 96.8 & 0.9 & 3.8 & 6.3 & 7.2 & 7.4 & 0.88 & 0.86 \\
\hline ITF172 $\times 65 \times 13-\mathrm{t} 1.3 \mathrm{MN} 120 \mathrm{AOFR}$ & 134.0 & 94.5 & 0.7 & 3.9 & 7.1 & 7.2 & 7.4 & 0.98 & 0.96 \\
\hline ITF202 $\times 65 \times 13-\mathrm{t} 1.4 \mathrm{MN} 150 \mathrm{AOFR}$ & 137.4 & 103.4 & 0.8 & 3.4 & 8.4 & 9.6 & 9.8 & 0.88 & 0.86 \\
\hline Mean, $P_{m}$ & & & & & & & & 0.94 & 0.93 \\
\hline Coefficient of variation, $V_{p}$ & & & & & & & & 0.08 & 0.08 \\
\hline Reliability index, $\beta$ & & & & & & & & 2.22 & 2.18 \\
\hline Resistance factor, $\phi$ & & & & & & & & 0.90 & 0.90 \\
\hline
\end{tabular}


Table 6

Comparison of experimental and numerical results with design strength for ETF and ITF loading condition under flanges fastened case.

\begin{tabular}{|c|c|c|c|c|c|c|c|c|c|c|c|}
\hline \multirow[t]{2}{*}{ Specimen } & \multirow[t]{2}{*}{$h / t$} & \multirow[t]{2}{*}{$N / t$} & \multirow[t]{2}{*}{$N / h$} & \multirow[t]{2}{*}{$r_{i} / t$} & \multirow{2}{*}{$\begin{array}{l}\text { Failure load } \\
\left(P_{\exp } \text { and } P_{\mathrm{FEA}}\right) \\
P \\
(\mathrm{kN})\end{array}$} & \multicolumn{3}{|c|}{$\begin{array}{l}\text { Web crippling strength predicted from } \\
\text { current design codes }\end{array}$} & \multicolumn{3}{|c|}{ Comparison } \\
\hline & & & & & & $\begin{array}{l}P_{\mathrm{BS}} \\
(\mathrm{kN})\end{array}$ & $\begin{array}{l}P_{\mathrm{EC}} \\
(\mathrm{kN})\end{array}$ & $\begin{array}{l}P_{\mathrm{NAS}} \\
(\mathrm{kN})\end{array}$ & $P / P_{\mathrm{BS}}$ & $P / P_{\mathrm{EC} 3}$ & $P / P_{\mathrm{NAS}}$ \\
\hline ETF142 × $60 \times 13-\mathrm{t} 1.3 \mathrm{MN} 90 \mathrm{A0FX}$ & 113.3 & 72.6 & 0.6 & 3.8 & 3.8 & 2.3 & 2.3 & 4.4 & 1.64 & 1.64 & 0.86 \\
\hline ETF142 × $60 \times 13-\mathrm{t} 1.3 \mathrm{MN} 120 \mathrm{AOFX}$ & 113.3 & 96.8 & 0.9 & 3.8 & 4.1 & 2.6 & 2.6 & 4.7 & 1.56 & 1.56 & 0.86 \\
\hline ETF172 $\times 65 \times 13-t 1.3 M N 120$ AOFX & 134.0 & 94.5 & 0.7 & 3.9 & 4.2 & 2.4 & 2.4 & 5.2 & 1.74 & 1.75 & 0.80 \\
\hline ETF202 $\times 65 \times 13-\mathrm{t} 1.4 \mathrm{MN} 120 \mathrm{AOFX}$ & 137.4 & 82.8 & 0.6 & 3.4 & 5.2 & 3.3 & 3.3 & 6.3 & 1.58 & 1.58 & 0.83 \\
\hline ETF202 $\times 65 \times 13-\mathrm{t} 1.4 \mathrm{MN} 150 \mathrm{AOFX}$ & 137.4 & 103.4 & 0.8 & 3.4 & 5.8 & 3.7 & 3.7 & 6.7 & 1.58 & 1.58 & 0.87 \\
\hline ETF202 $\times 65 \times 13-\mathrm{t} 1.4 \mathrm{MN} 100 \mathrm{AOFX}$ & 142.3 & 71.4 & 0.5 & 3.6 & 5.4 & 2.8 & 2.8 & 5.5 & 1.96 & 1.96 & 0.98 \\
\hline ETF202 $\times 65 \times 13-t 1.4$ MN150A0FX & 142.3 & 107.1 & 0.8 & 3.6 & 6.0 & 3.3 & 3.3 & 6.1 & 1.80 & 1.80 & 0.98 \\
\hline ETF202 $\times 65 \times 13-\mathrm{t} 1.4 \mathrm{MN} 200 \mathrm{AOFX}$ & 142.3 & 142.9 & 1.0 & 3.6 & 7.2 & 3.9 & 3.9 & 6.7 & 1.84 & 1.84 & 1.08 \\
\hline ITF142 × $60 \times 13-t 1.3$ MN90AOFX & 113.3 & 72.6 & 0.6 & 3.8 & 9.0 & 7.0 & 7.2 & 12.7 & 1.28 & 1.25 & 0.71 \\
\hline ITF142 × $60 \times 13-t 1.3 \mathrm{MN} 120 \mathrm{AOFX}$ & 113.3 & 96.8 & 0.9 & 3.8 & 9.4 & 7.2 & 7.4 & 13.5 & 1.31 & 1.28 & 0.70 \\
\hline ITF172 × $65 \times 13-t 1.3 \mathrm{MN} 120 \mathrm{AOFX}$ & 134.0 & 94.5 & 0.7 & 3.9 & 10.7 & 7.2 & 7.4 & 15.7 & 1.49 & 1.45 & 0.68 \\
\hline ITF202 $\times 65 \times 13-t 1.4 \mathrm{MN} 150$ A0FX & 137.4 & 103.4 & 0.8 & 3.4 & 13.5 & 9.6 & 9.8 & 20.3 & 1.41 & 1.38 & 0.67 \\
\hline Mean, $P_{m}$ & & & & & & & & & 1.60 & 1.59 & 0.83 \\
\hline Coefficient of variation, $V_{p}$ & & & & & & & & & 0.09 & 0.09 & 0.12 \\
\hline Reliability index, $\beta$ & & & & & & & & & 4.33 & 4.30 & 1.67 \\
\hline Resistance factor, $\phi$ & & & & & & & & & 0.90 & 0.90 & 0.90 \\
\hline
\end{tabular}
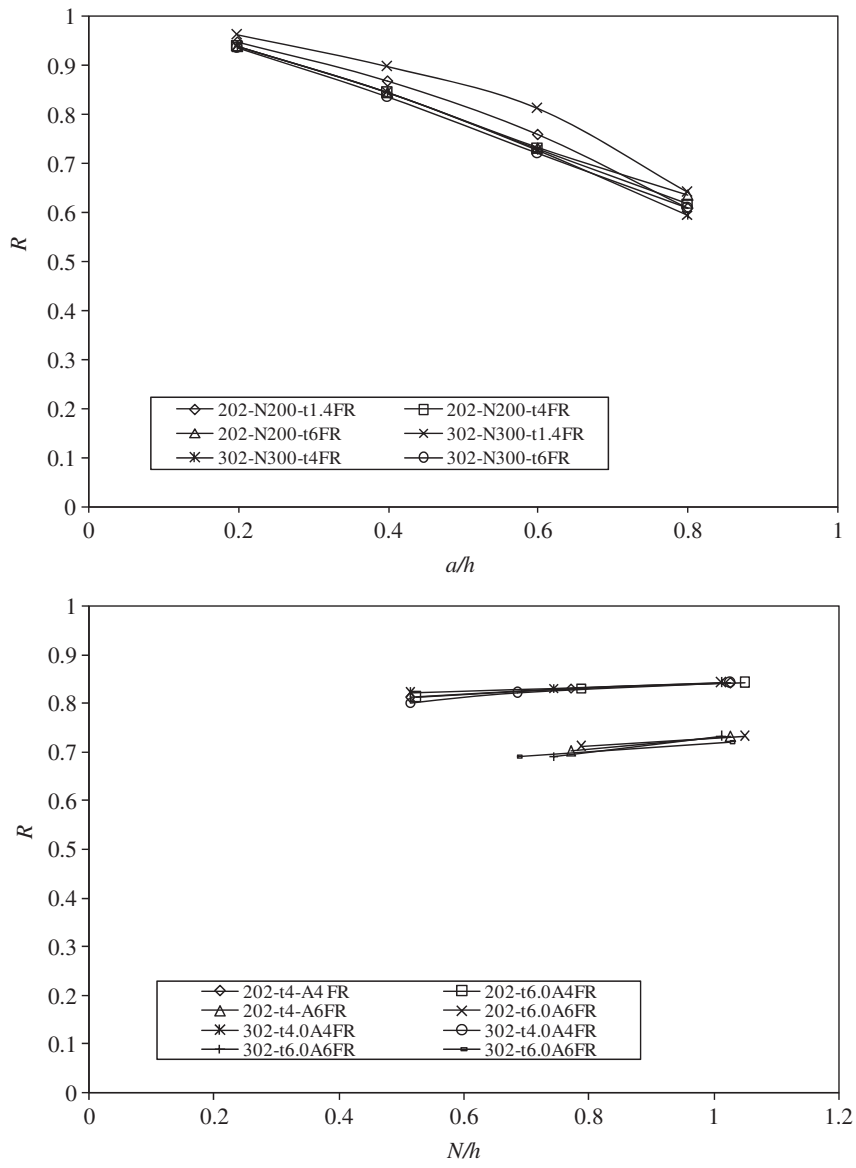

Fig. 3. Variation in reduction factors with $a / h$ and $N / h$ for flanges unfastened under ITF loading condition.

equal to $100 \mathrm{~mm}, 150 \mathrm{~mm}, 200 \mathrm{~mm}, 220 \mathrm{~mm}$, and $300 \mathrm{~mm}$ were used. For each series of specimens, the web crippling strengths of the sections without the web holes were obtained. Thus, the ratio of the web crippling strengths for sections with web holes divided by the sections without the web holes, which is the strength reduction factor $(R)$, was used to quantify the degrading influence of the web holes on the web crippling strengths. In Tables 1-4, the specimens were labelled such that the loading condition, the
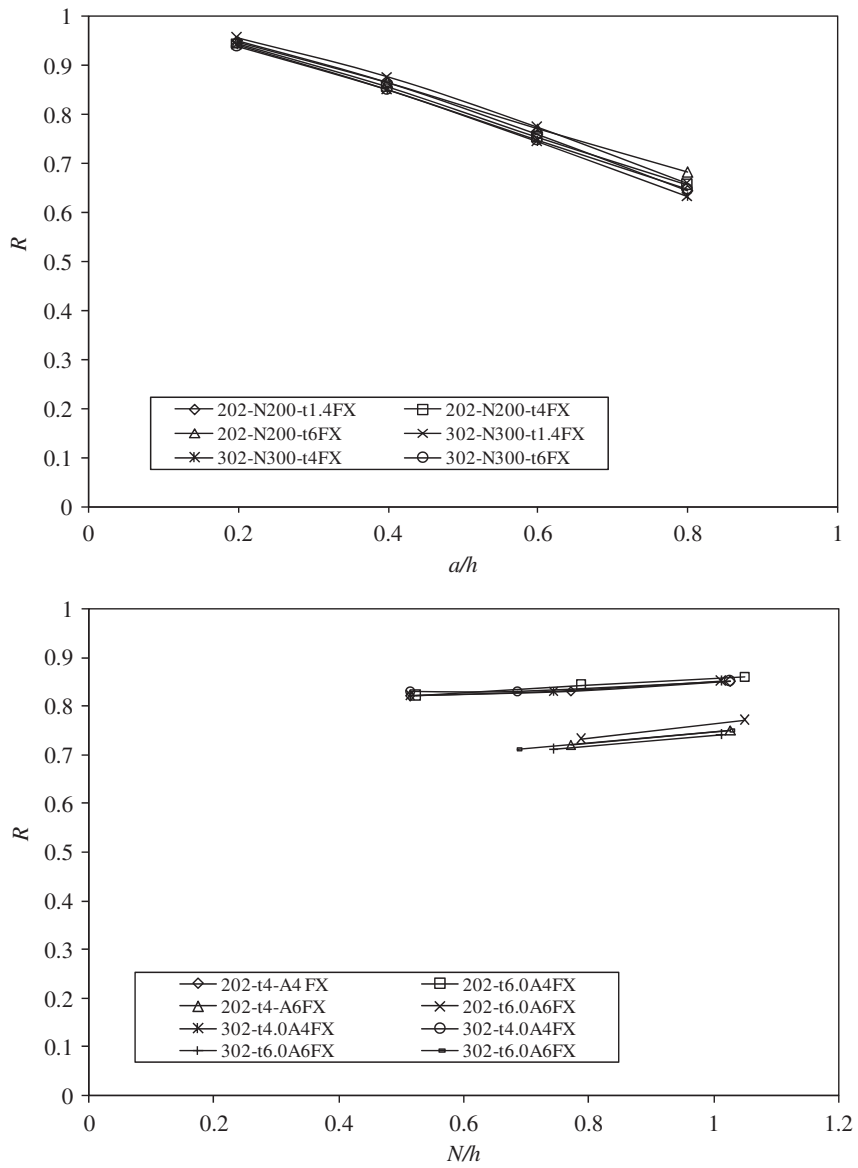

Fig. 4. Variation in reduction factors with $a / h$ and $N / h$ for flanges fastened under ITF loading condition.

nominal dimension of the specimen and the length of the bearing as well as the ratio of the diameter of the holes to the depth of the flat portion of the webs $(a / h)$ could be identified from the label. Details of the specimens labelling is described in Uzzaman et al. [8].

For the ITF loading conditions, a total of 144 specimens was analysed in the parametric study investigating the effect of the ratio $a / h$ and $N / h$. The cross-section dimensions as well as the web 

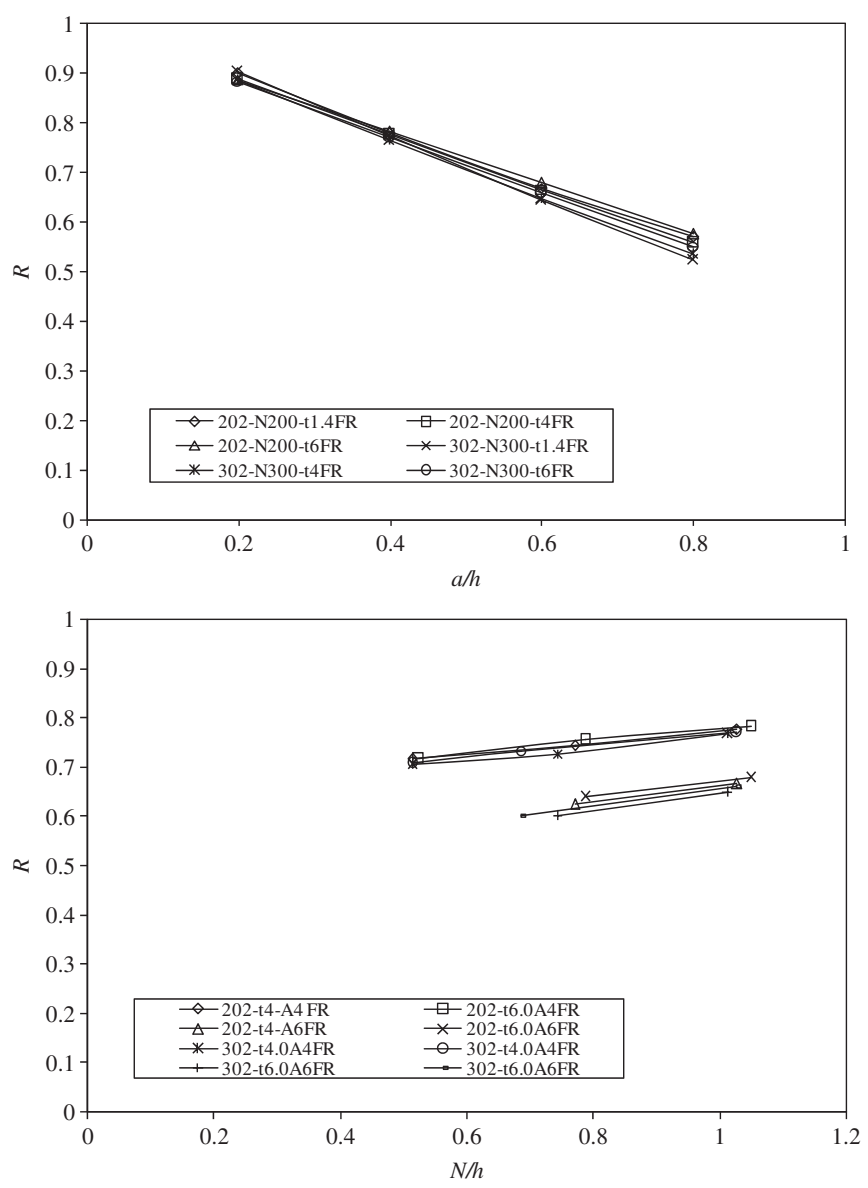

Fig. 5. Variation in reduction factors with $a / h$ and $N / h$ for flanges unfastened under ETF loading condition.

crippling strengths $\left(P_{\mathrm{FEA}}\right)$ per web predicted from the FEA are summarised in Tables 1 and 2 for flanges unfastened and fastened condition, respectively. The effect of $a / h$ and $N / h$ ratio on the web crippling strength on the reduction factor for flanges unfastened and fastened condition are shown in Figs. 3 and 4 for C202 and C302 specimens, respectively. It is seen from these graphs that the parameter $a / h$ and $N / h$ noticeably affects the web crippling strength and the reduction factor.

For the ETF loading condition, a total of 144 specimens was analysed in the parametric study investigating the effect of the ratio $a / h$ and $N / h$. The cross-section dimensions as well as the web crippling strengths $\left(P_{\mathrm{FEA}}\right)$ per web predicted from the FEA are summarised in Tables 3 and 4 for flanges unfastened and fastened condition, respectively. The effect of $a / h$ and $N / h$ on the web crippling strength on the reduction factor for flanges unfastened and fastened condition are shown in Figs. 5 and 6 for C202 and C302 specimens, respectively. It is seen from these graphs that the parameter $a / h$ and $N / h$ noticeably affects the web crippling strength and the reduction factor.

\section{Reliability analysis}

The reliability of the cold-formed steel section design rules is evaluated using reliability analysis. The reliability index $(\beta)$ is a relative measure of the safety of the design. A target reliability index of 2.5 for cold-formed steel structural members is recommended as a lower limit in the NAS Specification [1]. The design rules are considered to be reliable if the reliability index is greater than or equal to 2.5 . The load combination of $1.2 \mathrm{DL}+1.6 \mathrm{LL}$ as
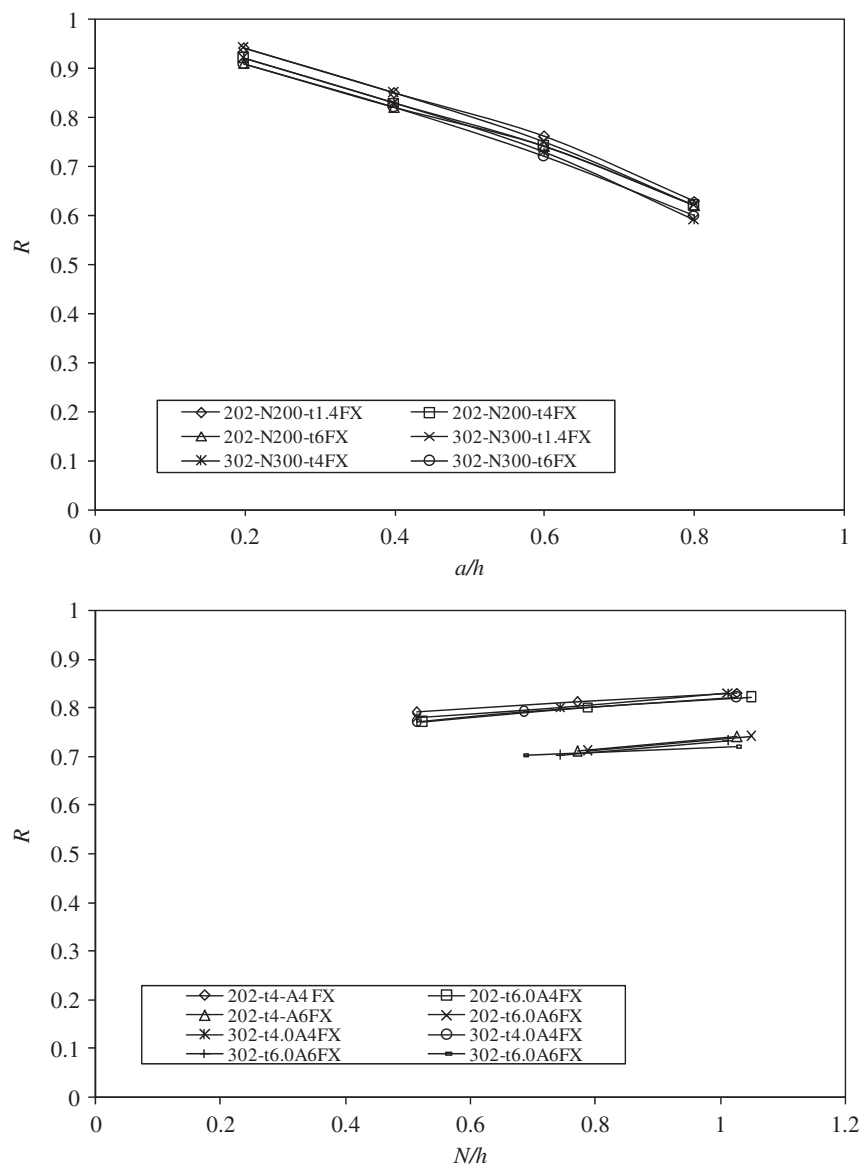

Fig. 6. Variation in reduction factors with $a / h$ and $N / h$ for flanges fastened under ETF loading condition.

Table 7

Statistical analysis for comparison of strength reduction factor for flanges unfastened under ITF loading condition.

\begin{tabular}{ll}
\hline Statistical parameters & $R($ Test and FEA $) / R_{p}$ \\
& $(1.05-0.54(a / h)+0.01(N / h))$ \\
\hline Mean, $P_{m}$ & 1.01 \\
Coefficient of variation, $V_{p}$ & 0.04 \\
Reliability index, $\beta$ & 2.63 \\
Resistance factor, $\phi$ & 0.90 \\
\hline
\end{tabular}

Table 8

Statistical analysis for comparison of strength reduction factor for flanges fastened under ITF condition.

\begin{tabular}{ll}
\hline Statistical parameters & $R($ Test and FEA $) / R_{p}$ \\
& $(1.01-0.51(a / h)+0.06(N / h))$ \\
\hline Mean, $P_{m}$ & 1.00 \\
Coefficient of variation, $V_{p}$ & 0.03 \\
Reliability index, $\beta$ & 2.62 \\
Resistance factor, $\phi$ & 0.90 \\
\hline
\end{tabular}

specified in the American Society of Civil Engineers Standard [10] was used in the reliability analysis, where DL is the dead load and LL is the live load. The statistical parameters are obtained from Table F1 of the NAS Specification [1] for compression members, where $M_{m}=1.10, F_{m}=1.00, V_{M}=0.10$, and $V_{F}=0.05$, which are the 
Table 9

Statistical analysis for comparison of strength reduction factor for flanges unfastened under ETF loading condition.

\begin{tabular}{ll}
\hline Statistical parameters & $\begin{array}{l}R(\text { Test and FEA }) / R_{p} \\
(0.90-0.60(a / h)+0.12(N / h))\end{array}$ \\
\hline Mean, $P_{m}$ & 1.00 \\
Coefficient of variation, $V_{p}$ & 0.03 \\
Reliability index, $\beta$ & 2.61 \\
Resistance factor, $\phi$ & 0.90 \\
\hline
\end{tabular}

Table 10

Statistical analysis for comparison of strength reduction factor for flanges fastened under ETF condition.

\begin{tabular}{ll}
\hline Statistical parameters & $R($ Test and FEA $) / R_{p}$ \\
& $(0.95-0.50(a / h)+0.08(N / h))$ \\
\hline Mean, $P_{m}$ & 1.00 \\
Coefficient of variation, $V_{p}$ & 0.02 \\
Reliability index, $\beta$ & 2.62 \\
Resistance factor, $\phi$ & 0.90 \\
\hline
\end{tabular}

mean values and coefficients of variation for material properties and fabrication factors.

The statistical parameters $P_{m}$ and $V_{P}$ are the mean value and coefficient of variation of load ratio are shown in Tables 7-10, respectively. In calculating the reliability index, the correction factor in the NAS Specification was used. Reliability analysis is detailed in the NAS Specification [1]. In the reliability analysis, a constant resistance factor $(\phi)$ of 0.90 was used. It is shown that the reliability index $(\beta)$ is greater than the target value of 2.5 as shown in Tables 7-10.

\section{Comparison of experimental and numerical results with current design strengths for cold-formed steel sections without web holes}

As mentioned earlier, the current cold-formed design standards $([1,11,12])$ do not provide design rules for cold-formed steel sections with web holes subjected to web crippling under ITF and ETF loading conditions. The web crippling strength predicted from tests and FEA results were compared with the web crippling strength obtained from design codes. According to Beshara and Schuster [13], NAS Specification [1] design expressions has parameter ranges. In the design equations for the case of flanges fastened to the support, the specimen thicknesses ranged from $1.16 \mathrm{~mm}$ to $1.45 \mathrm{~mm}$ and the yield stresses ranged from $323 \mathrm{MPa}$ to $448 \mathrm{MPa}$. The design equations for the case of flanges unfastened to the support, specimen thicknesses ranged from $1.194 \mathrm{~mm}$ to $1.326 \mathrm{~mm}$ and yield stress ranged $301.8 \mathrm{MPa}$ to 324.6 MPa. However, it is noted, the above range of specimens were considered for the results comparison.

In the case of flanges unfastened, Table 5 shows the comparison of web crippling strength with design strength for ETF and ITF loading condition. The NAS Specification design strength was not considered as the $r_{i} / t$ ratio limit was greater than three. In the British Standard and Eurocode comparison, the mean values of ratio are 0.94 and 0.93 with the corresponding coefficients if variation (COV) of 0.08 and 0.08 , and the reliability indices $(\beta)$ of 2.22 and 2.18 , respectively.

In the case of flanges fastened, Table 6 shows the comparison of web crippling strength with design strength for ETF and ITF loading condition. British Standards and Eurocodes provides unreliable web crippling strengths predictions for the flanges fastened case. A comparison of these values with the corresponding experimental and numerical values indicates that although the British Standard and Eurocode values are lower bound, that they are about $60 \%$ lower than the experimental and numerical failure loads. For the NAS Specification comparison, the mean values of ratio are 0.83 with the corresponding coefficients of variation (COV) of 0.12 and the reliability indices $(\beta)$ of 1.67 . It is noted that for the unfastened case agreement is very good, however, for the fastened case, the comparison is less reliable due to the post buckling strength effect not being fully considered in the design codes.

\section{Proposed strength reduction factors}

Comparing the failure loads of the channel sections, with and without web holes as shown in Tables $1-4$, it can be seen that, as expected, the failure load decreases as the size of the web holes increases and the failure load increases as the length of the bearing plates decreases. Evaluation of the experimental and the numerical results shows that the ratios $a / h$ and $N / h$ are the primary parameters influencing the web crippling behaviour of the sections with web holes.

\subsection{Proposed strength reduction factor ITF loading condition}

Based on both the experimental study reported by Uzzaman et al. [8] and the numerical results obtained from this study, two strength reduction factor $\left(R_{p}\right)$ are proposed using bivariate linear regression analysis for the ITF loading condition. For the flanges
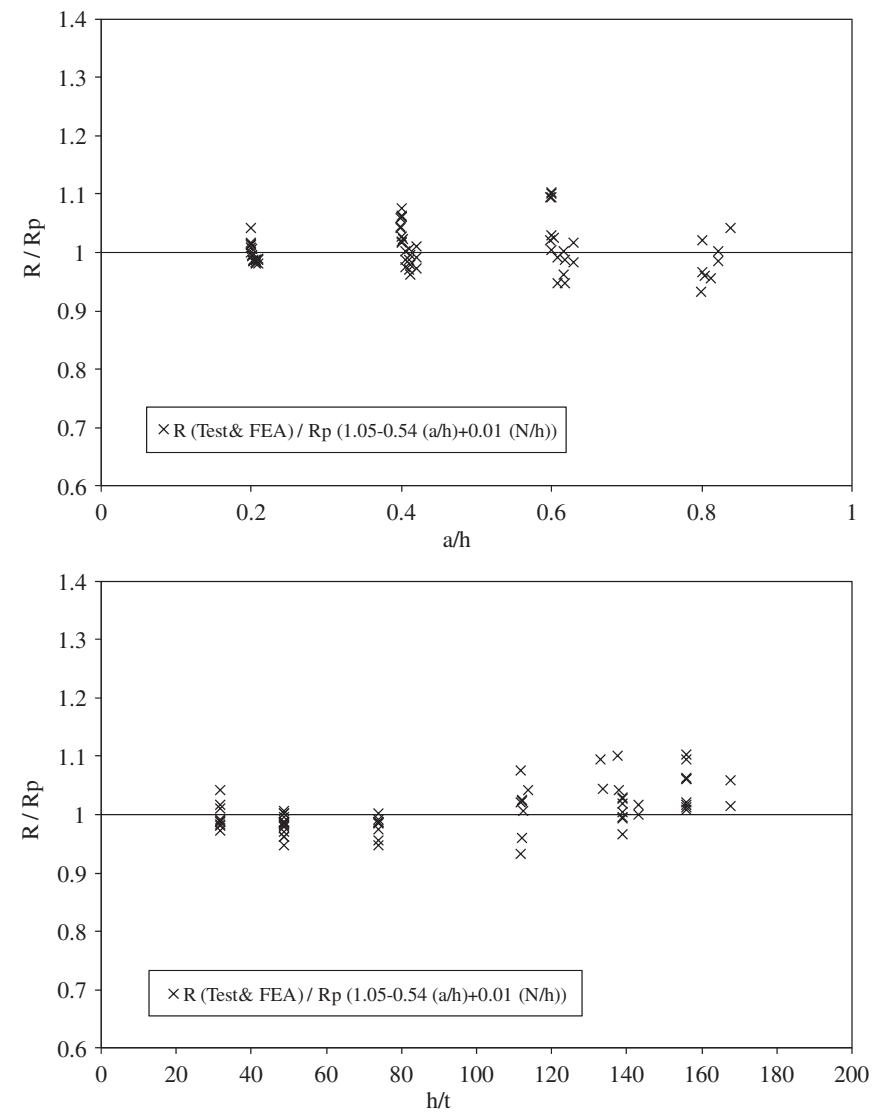

Fig. 7. Comparison of strength reduction factor for flanges unfastened condition under ITF loading condition. 
unfastened condition;

$R_{P}=1.05-0.54\left(\frac{a}{h}\right)+0.01\left(\frac{N}{h}\right) \leq 1$

For the flanges fastened condition;

$R_{P}=1.01-0.51\left(\frac{a}{h}\right)+0.06\left(\frac{N}{h}\right) \leq 1$

The limits for the reduction factor Eqs. (1) and (2) are $h / t \leq 156, N / t \leq 84, N / h \leq 0.63, a / h \leq 0.8$, and $\theta=90^{\circ}$.

\subsection{Proposed strength reduction factor ETF loading condition}

Based on both the experimental study reported by Uzzaman et al. [8] and the numerical results obtained from this study, two strength reduction factor $\left(R_{p}\right)$ are proposed using bivariate linear regression analysis for the ETF loading condition.

For the flanges unfastened condition;

$R_{P}=0.90-0.60\left(\frac{a}{h}\right)+0.12\left(\frac{N}{h}\right) \leq 1$

For the flanges fastened condition;

$R_{P}=0.95-0.50\left(\frac{a}{h}\right)+0.08\left(\frac{N}{h}\right) \leq 1$

The limits for the reduction factor Eqs. (1) and (2) are $h / t \leq 156, N / t \leq 84, N / h \leq 0.63, a / h \leq 0.8$, and $\theta=90^{\circ}$.
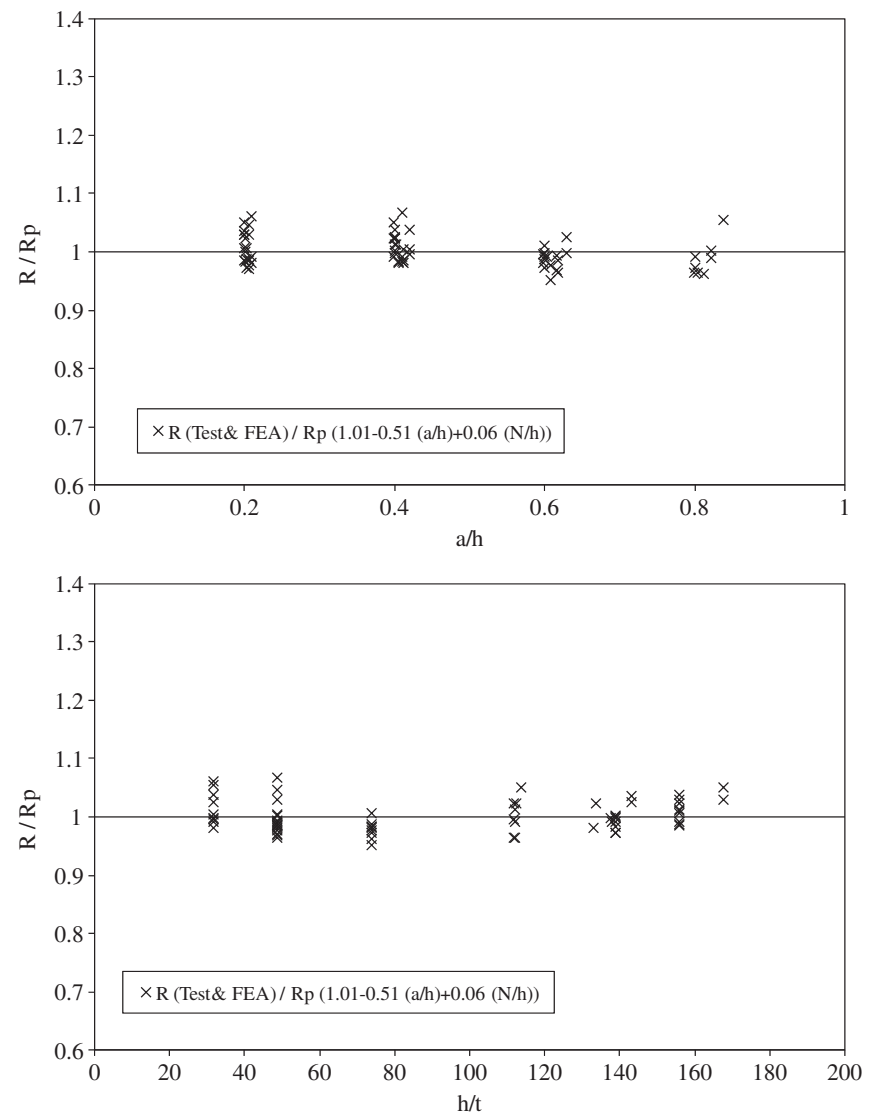

Fig. 8. Comparison of strength reduction factor for flanges fastened condition under ITF loading condition.

\section{Comparison of the experimental and numerical results with the proposed reduction factor}

For the ITF loading condition, the values of the strength reduction factor $(R)$ obtained from the experimental and the numerical results are compared with the values of the proposed strength reduction factor $\left(R_{p}\right)$ calculated using Eqs. (1) and (2), as plotted against the ratios $a / h$ and $h / t$ in Figs. 7 and 8 for flanges unfastened and fastened cases, respectively. Tables 7 and 8 summarises a statistical analysis to define the accuracy of the proposed design equations. The values of the proposed reduction factor are generally conservative and agree well with the experimental and the numerical results for ITF loading conditions. As can be seen, the proposed reduction factors are generally conservative and agree with the experimental and the numerical results for both load cases. The mean value of the web crippling reduction factor ratio are 1.01 and 1.00 with the corresponding COV of 0.04 and 0.03 , and reliability index $(\beta)$ of 2.63 and 2.62 for the flanges are unfastened and fastened conditions, respectively. Thus, the proposed strength reduction factor equations are able to predict the influence of the web holes on the web crippling strengths of channel sections for the ITF loading condition.

For the ETF loading condition, the values of the strength reduction factor $(R)$ obtained from the experimental and the numerical results are compared with the values of the proposed strength reduction factor $\left(R_{p}\right)$ calculated using Eqs. (3) and (4), as plotted against the ratios $a / h$ and $h / t$ in Figs. 9 and 10 for flanges unfastened and fastened cases, respectively. Tables 9 and 10 summarise a statistical analysis to define the accuracy of the proposed design equations. The values of the proposed reduction factor are generally conservative and agree well with the experimental and the numerical results for the ETF loading conditions. As can be seen, the proposed reduction factors are generally
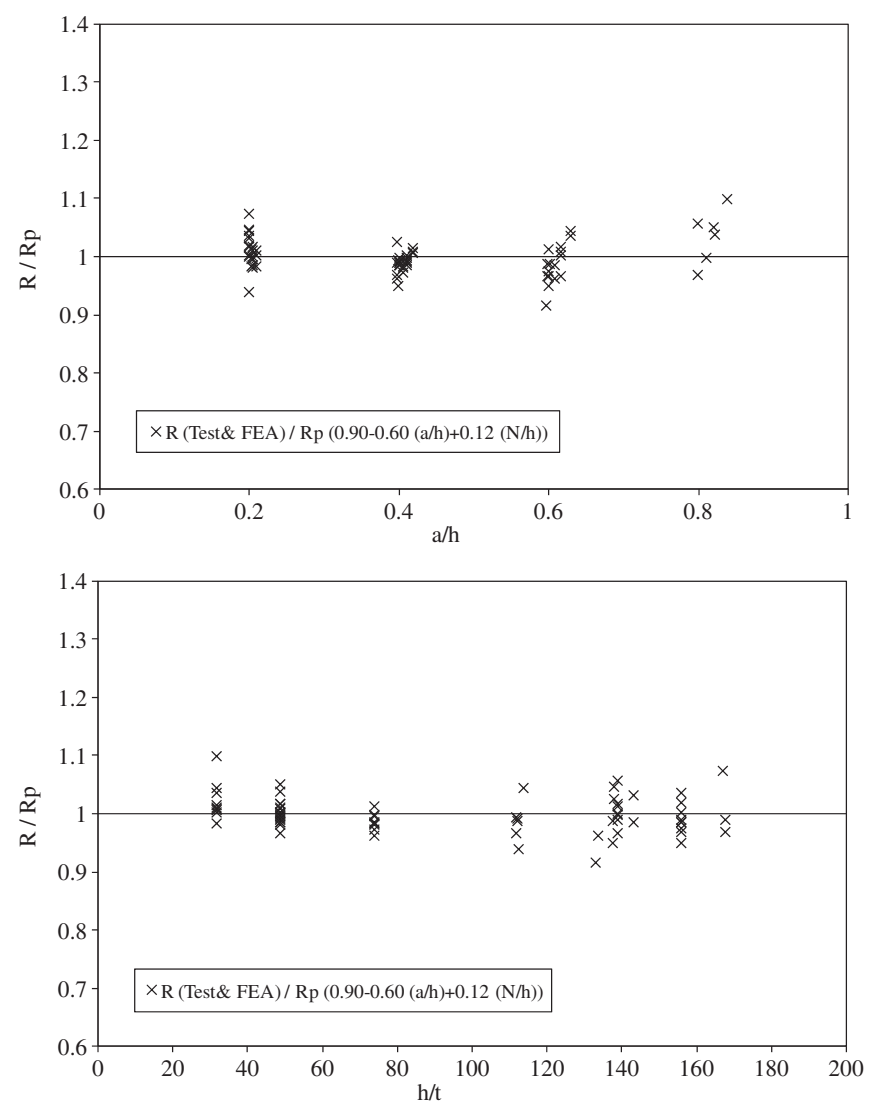

Fig. 9. Comparison of strength reduction factor for flanges unfastened condition under ETF loading condition. 

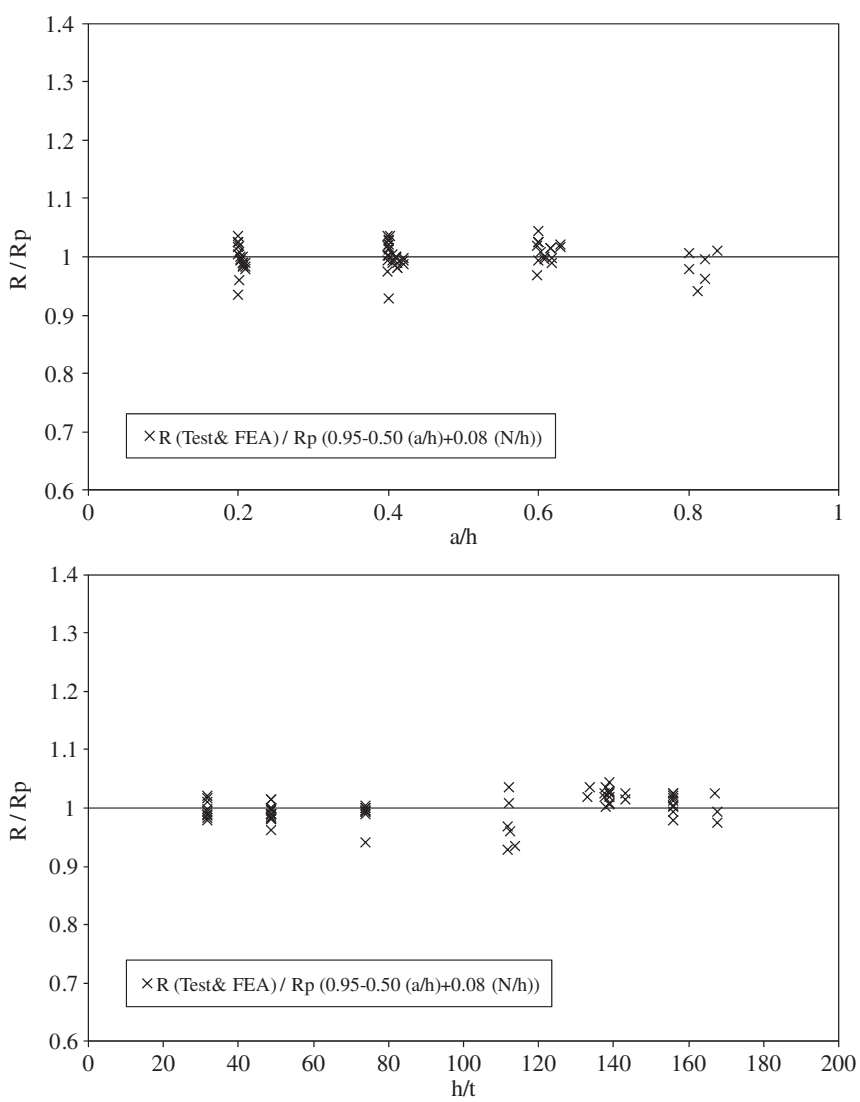

Fig. 10. Comparison of strength reduction factor for flanges fastened condition under ETF loading condition.

conservative and agree with the experimental and the numerical results for both load cases. The mean value of the web crippling reduction factor ratio are 1.00 and 1.00 with the corresponding COV of 0.03 and 0.02 , and reliability index $(\beta)$ of 2.61 and 2.62 for the flanges are unfastened and fastened conditions, respectively. Thus, the proposed strength reduction factor equations are able to predict the influence of the web holes on the web crippling strengths of channel sections for the ITF loading condition.

\section{Conclusions}

A parametric study of lipped channel sections with circular web holes subjected to web crippling has been presented. The web holes are located at the mid-depth of the webs and centred beneath the bearing plates. A non-linear finite element model was used in the parametric study, which has been verified against experimental test results. Evaluation of the experimental and the numerical results show that the ratios $a / h$ and $N / h$ are the primary parameters influencing the web crippling behaviour of the sections with web holes. In order to determine the effect of the ratios $a / h$ and $N / h$ on the web crippling strength, parametric studies were carried out considering the web holes, the crosssection sizes and the different bearing plate lengths.

Based on the available data obtained from the experimental and numerical investigations, web crippling strength reduction factor equations were proposed for the ITF and ETF loading conditions for the cases of both flanges unfastened and fastened to the support. Reliability analysis was performed to evaluate the reliability of the proposed strength reduction factors. It was shown that the proposed strength reduction factors are generally conservative and agree well with the experimental and the numerical results. The proposed strength reduction factors are capable of producing reliable limit state design when calibrated with the resistance factor of $0.90(\phi=0.90)$.

\section{Acknowledgements}

The authors gratefully acknowledge the help given by Metsec $\mathrm{Plc}$, UK, in supplying the materials. The authors also wish to thank $\mathrm{Mr}$ Chris Cameron and Mr Andrew Crockett for their assistant in preparing the specimens and carrying out the experimental testing.

\section{References}

[1] N.A.S., North American Specification for the Design of Cold-Formed Steel Structural Members, American Iron and Steel Institute, AISI S100-2007, AISI Standard, 2007.

[2] Yu WW, Davis CS. Cold-formed steel members with perforated elements. Journal of the Structural Division 1973;99:2061-77.

[3] Sivakumaran KS, Zielonka KM. Web crippling strength of thin-walled steel members with web opening. Thin-Walled Structures 1989;8:295-319.

[4] LaBoube RA, Yu WW, Deshmukh SU, Uphoff CA. Crippling capacity of web elements with openings. Journal of Structural Engineering 1999;125:137-41.

[5] Chung KF. Structural perfomance of cold formed sections with single and multiple web openimgs. (Part-1 experimental investigation). The Structural Engineer 1995;73.

[6] Uzzaman A, Lim JBP, Nash D, Rhodes J, Young B. Web crippling behaviour of cold-formed steel channel sections with offset web holes subjected to interior-two-flange loading. Thin-Walled Structures 2012;50:76-86.

[7] Zhou F, Young B. Web crippling of aluminium tubes with perforated webs. Engineering Structures 2010;32:1397-410.

[8] Uzzaman A, Lim JBP, Nash D, Rhodes J, Young B, Cold-Formed Steel Sections With Web Openings Subjected To Web Crippling Under Interior-Two-Flange And End-Two-Flange Loading Conditions-Part I: tests And Finite Element Analysis, Thin-Walled Structures (reference to corresponding paper in the same issue).

[9] ANSYS, User's manual, revision 11.0, in, Swanson Analysis System, 2011.

[10] ASCE, Minimum design loads for buildings and other structures, in, American Society of Civil Engineers Standard, New York, 2005.

[11] BS5950, Structural use of steelwork in buildings, in: Part 5 code of practice for the design of cold-formed sections, British Standards Institution, London, 1998.

[12] Eurocode-3, Design of steel structures: Part 1.3: general rules - supplementary rules for cold-formed thin gauge members and sheeting, in: ENV 19931-3, European Committee for Standardization, Brussels, Belgium, 1996.

[13] B. Beshara, R.M. Schuster, Web crippling data and calibations of cold formed steel members, in, Uinversity of Waterloo, Canada, 2000. 